\title{
Researches on Crack Propagation of the Two Filled Noncoalescent Coplanar Flaws under the High Strain Rate Loading by means of AUTODYN-Based Simulation
}

\author{
Guangming Zhao $\mathbb{D}^{1,2}$ Chongyan Liu $\mathbb{D}^{1,2}$ Cheng Pan, ${ }^{3}$ and Xiangrui Meng ${ }^{1,2}$ \\ ${ }^{1}$ State Key Laboratory of Mining Response and Disaster Prevention and Control in Deep Coal Mines, Anhui University of Science \\ and Technology, Huainan 232001, China \\ ${ }^{2}$ School of Mining and Safety Engineering, Anhui University of Science and Technology, Huainan 232001, China \\ ${ }^{3}$ School of Civil Engineering, Southeast University, Nanjing, Jiangsu 210096, China
}

Correspondence should be addressed to Chongyan Liu; chongyanliu@126.com

Received 27 July 2020; Accepted 18 November 2020; Published 24 November 2020

Academic Editor: Zhijie Wen

Copyright (c) 2020 Guangming Zhao et al. This is an open access article distributed under the Creative Commons Attribution License, which permits unrestricted use, distribution, and reproduction in any medium, provided the original work is properly cited.

To study the influence of fillings on rock failure. By turning to the Drucker-Prager strength model and cumulative damage criteria, investigations are made, with the nonlinear AUTODYN software, into crack propagation behaviors in crack-filled and unfilled specimens under uniaxial dynamic loading. Under investigations are crack initiation position, sequence and angle, and coalescence mode. According to the mode of propagation and coalescence, cracks are divided into three types, i.e., the tensile wing, the antiwing, and the horsetail. The simulation results show that under uniaxial dynamic loading, differences are found in initiation position, angle, and coalescence mode for specimens with cracks filled or otherwise. However, filling does not affect crack initiation sequence. Under the same loading, the damage to filled specimen is less severe than that to the unfilled specimen.

\section{Introduction}

Deep underground civil engineering construction such as energy resources exploration and subway construction have given rise to large scale research done on in-depth rock mechanics $[1,2]$. Underground rock masses contain a large number of structural planes such as cracks, joints, and bedding planes with different sizes, which are frequently affected by tectonic strain and engineering disturbance [3]. Consequently, the mechanical property and the stability of the rock strata are inevitably influenced. As demonstrated by numerous underground engineering practices, the imposition of a load results in internal joint crack propagation and coalescence, leading to the destabilization and damage of rock masses [4]. To obtain a clear understanding, many scholars have conducted physical experiments [5-7] and numerical simulation [8-11] to investigate the evolution process of crack initiation, propagation, and coalescence. Generally, it is easier to obtain the cracking behavior by numerical simulation than by physical means. For example, by adopting Rock Failure Process Analysis System (RFPA), Wang et al. [8] conducted a numerical simulation experiment with the double flaw rock specimen under uniaxial compression and simulated the complete breaking process of concrete samples from crack initiation, through propagation and macrocrack formation. By adopting the nonlinear dynamic software AUTODYN, Li and Wong [9] clarified 11 modes of coalescence of the two crack rocks and classified 11 modes into tensile, shear, and tensile-shear coalescence in accordance with the crack-inducing mechanism.

Rock mass is often under dynamic loading induced by blasting or earthquake. In this case, the rocks always experience an internal cracking process through the stages of crack initiation, propagation, and coalescence [12]. In blasting, the loading strain rate ranges within $10^{-4} \sim 10^{3} \mathrm{~s}^{-1}$. It is generally believed that the strain rate less than $10^{-4} \mathrm{~s}^{-1}$ can be classified as a low strain rate (induced by static loading); the strain rate ranges within $10^{-4} \sim 10^{2} \mathrm{~s}^{-1}$ constitutes the medium strain rate 
(induced by pseudodynamic loading), whereas the strain rate larger than $10^{2} \mathrm{~s}^{-1}$ can be divided into high strain rate (induced by dynamic loading) [13].

At present, researches on noncoalescent crack propagation mainly revolve around low and medium strained rock. For example, Zhang et al. [14] conducted investigations into the strength features of the noncoalescent rock and crack coalescence modes with specimens under the strains of 1.7 $\times 10^{-5} \mathrm{~s}^{-1}, 1.7 \times 10^{-3} \mathrm{~s}^{-1}$, and $1.7 \times 10^{-1} \mathrm{~s}^{-1}$. In addition, the specimens are with internal cracks at various positions and of various numbers. Zhang and Wong [15] adopted the adhesive particle model and numerical simulation to investigate individual crack specimens with certain inclination angles and under low or medium strains. Under low strained uniaxial loading, Lee and Jeon [16] conducted investigations into the stress-strain curve features of the noncoplanar crack of the rock body, and the factors under investigation include crack initiation, propagation, and integration. In addition, the crack initiation stress and coalescence stress of the double flaw specimen were analyzed and compared with the two stresses of the single flaw specimen. Up to now, researches on the rock mechanical behavior under high strains are mainly confined to undisturbed rocks. Therefore, it is necessary to conduct further investigations into the coalescence process under high strains.

Actually, there may often be fillings in the cracks. Under natural conditions, cracks may be filled with gravel. In the actual engineering process, they may be filled with such engineering substances as concrete. And the filling will reduce the stress concentration strength around the crack, and the researches available today show that the initial load for filled cracks is apparently higher than that for unfilled cracks and the initial cracking position is found moving nearer to the terminal [17].

Taking the above-mentioned researches into account, the nonlinear dynamic software AUTODYN is used as the research method, and the Drucker-Prager strength criterion and the cumulative damage (CD) criterion proposed by Persson are applied in the paper [18]. We will conduct investigations into the crack propagation and coalescence process of the two coplanar cracks of different inclination angles and under high straining loads. Meanwhile, the impact of fillings on rock failure is also considered, hoping to draw some beneficial conclusions and make significant contributions to the consolidation of the rocks under construction.

\section{Numerical Model}

2.1. The Calculation Model. For the simulation, a $150 \mathrm{~mm}$ $\times 75 \mathrm{~mm}$ planar model was adopted as shown in Figure 1 . In the model, there are two collinear joints with the length $2 a=12.7 \mathrm{~mm}$, the width $w=1.27 \mathrm{~mm}$, the rock bridge length $L=12.7 \mathrm{~mm}$, and the inclination angle $\beta$ is a variable ranging from $0^{\circ}$ to $90^{\circ}$. In the simulation, a load will be imposed on the top and on the bottom of the specimen with $\sigma_{\max }=40$ $\mathrm{MPa}$ and the loading rate was set as $80 \mathrm{MPa} / \mathrm{ms}$, as shown in Figure 2. As shown in Figure 3, under such loading conditions, the strain is imposed on the unfilled specimen along the $X$ direction and at the angle of $45^{\circ}$. It can be seen from

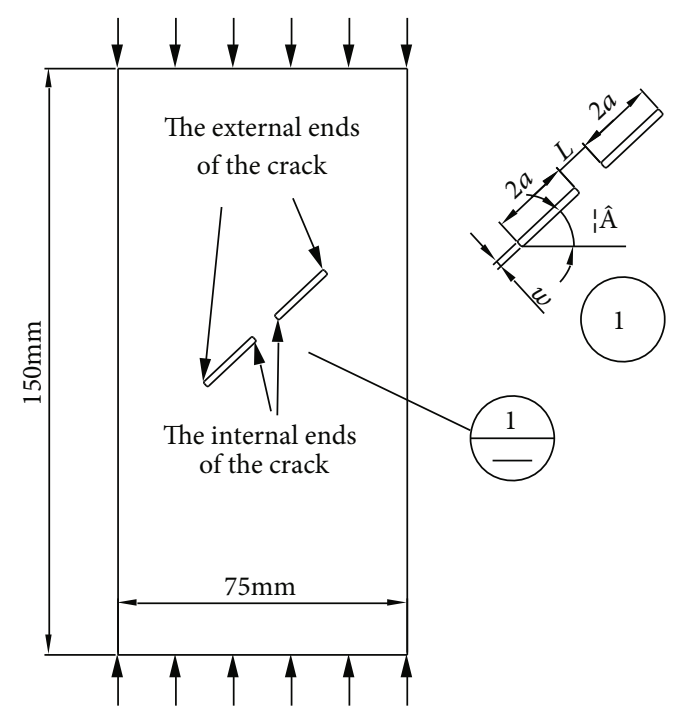

FIGURE 1: The schematic diagram of the calculation model.

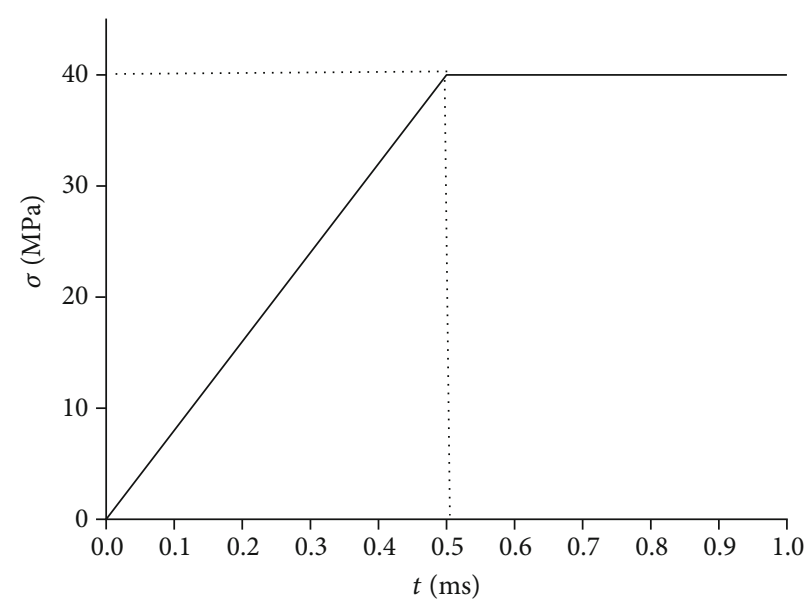

Figure 2: The loading conditions for the model.

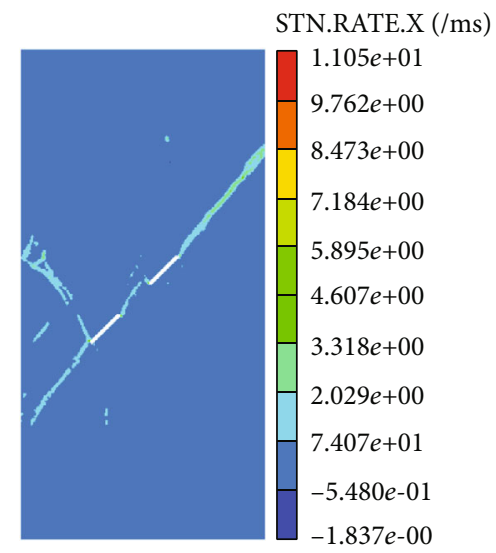

FIGURE 3: Direction and at the angle of $45^{\circ}(t=0.05 \mathrm{~ms})$.

the figure that along the horizontal direction, the strain on the specimen reached the order of magnitude of $10^{3} \mathrm{~s}^{-1}$.

2.2. The Material Model. The present numeral simulation employed two materials: the matrix rock and the concrete used 


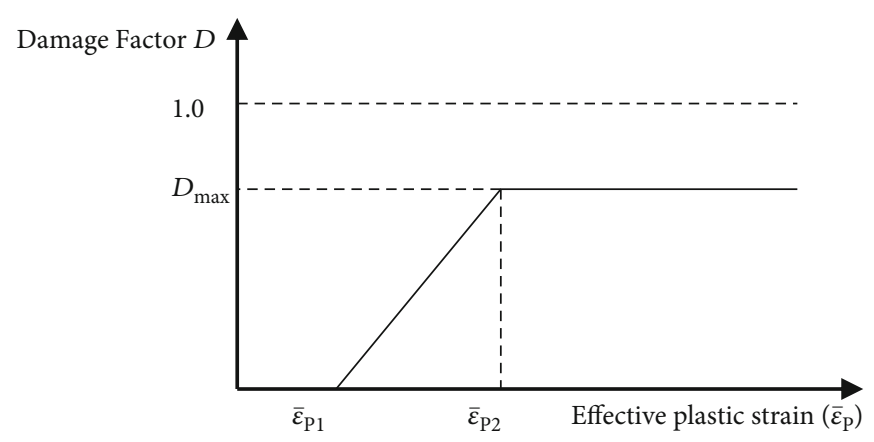

FIgURE 4: Curves depicting cumulative damage as a function of effective plastic strain.

as filling. For the concrete, the parameters in CONC-35MPA were directly selected from the AUTODYN material database. When it comes to the detailed introduction of the RiedelHiermaier-Thoma (RHT) model, please refer to Reference [19]. In what follows, we will focus on the description of the rock material model.

The constitutive model of the AUTODYN materials can be described as consisting of three parts: the state equation, the strength model, and the failure criterion. The state equation is used to solve the problems of volume deformation brought about by the pressure. According to Reference [20], the linear state equation can be selected to describe rock materials. The strength model is used to solve the problems of shape deformation brought about by the deviatoric stress. According to Reference [21], it would be proper to use the Drucker-Prager strength criterion as the strength constitutive. Taking into account the fact that the macrocrack formation is not an abrupt phenomenon but a damage cumulating process [9], the cumulative damage $(C D)$ was selected as the failure criterion.

By defining damage factor $D$, the CD failure criterion is determined and used to describe the strength decline in the gradual damage process of the material. The damage factor serves as a function of material deformation. When the effective plastic strain $\bar{\varepsilon}_{P}$ is below a certain value $\bar{\varepsilon}_{P 1}, D=0$; when it exceeds $\bar{\varepsilon}_{P 1}$, D will increase linearly to its maximum $D_{\max }$ $(<1)$. At this time, the corresponding effective plastic strain will be $\bar{\varepsilon}_{P 2}$, as shown in Figure 4 . The equation for $D$ will be

$$
D=D_{\max }\left(\frac{\bar{\varepsilon}_{P}-\bar{\varepsilon}_{P 1}}{\bar{\varepsilon}_{P 2}-\bar{\varepsilon}_{P 1}}\right) .
$$

In the process of calculation, the value of damage factor $D$ will be used to modify the material's volume modulus $K$, the shear modulus $G$, and the yielding strength $Y$. According to Reference [19], when the material is damaged most severely, the material under compression still retains certain strength residue. However, when the material is under the tensile strain, such residue cannot be retained. Therefore, the yielding strength is reduced in two ways. When the value of the static hydraulic pressure is positive, then

$$
Y_{\mathrm{dam}}=Y(1-D)
$$

When it is negative, then

$$
Y_{\mathrm{dam}}=Y\left(1-\frac{D}{D_{\max }}\right) .
$$

However, in the compressing process, the volume modulus and the shear modulus are not affected. But in the tensile process, when $D$ reaches $D_{\max }$, the volume modulus and the shear modulus will diminish to 0 in accordance with $1-D$ / $D_{\text {max }}$, as shown in Figure 5.

Wong et al. $[9,21]$ verified the rationale of the simulation of the crack propagation path by means of the DPCD model, showing that the derived path is in agreement with the experiment results. For the rock material, the present study will adopt the following dynamic parameters [9]: strength: $2.44 \mathrm{~g} / \mathrm{cm}^{3}$; plastic modulus: $51.92 \mathrm{GPa}$; Poisson ratios: 0.18; $\varepsilon_{p 1}=10^{-4} ; \varepsilon_{p 2}=10^{-3}$; and $D_{\max }=0.6$.

\section{The Numerical Simulation: Results and Analyses}

\subsection{Crack Typology and Identification}

3.1.1. The Basic Crack Types. Crack researches mainly revolve around the studies of its type, initiation position, propagation path, and coalescence mode. When it comes to underload crack propagation, Cen et al. [22] derived five basic modes. They pointed out the differences between the high-strained crack mode and the static loading crack mode. Furthermore, this research indicated the change of the secondary crack from the compound crack at the first stage to the tensile crack at the last stage. Wong and Einstein [5] found similar phenomena in their experiments. In previous studies, Li and Wong [9] took into account the crack's tensile/shear property and the propagation path and divided the basic crack types of the newly developed cracks into three: the tensile wing crack, the antiwing crack, and the horsetail crack (see Figure 6).

Through numerical simulation, we have also observed three types of crack, as shown in Figure 7. As can be seen from the figure, different cracks develop according to different initiation sequences. With unfilled joints, the wing crack appeared rather early and generally at the end of the joint. After initiation, the crack developed along a curved path 


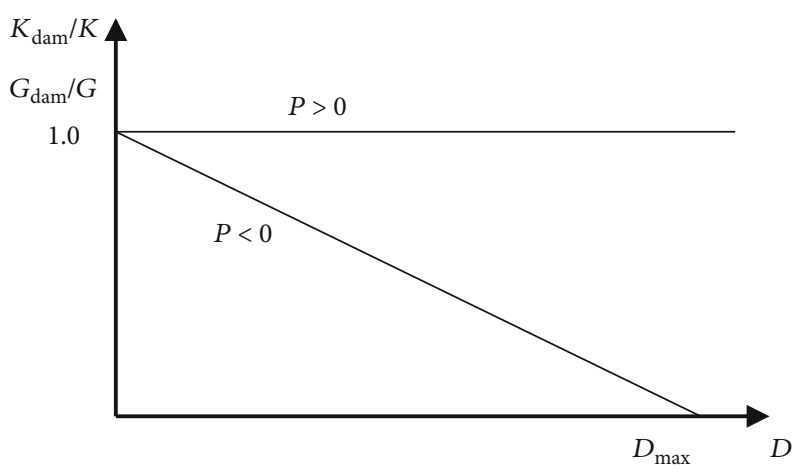

FIGURE 5: Curves depicting volume modulus and shear modulus as functions of damage.

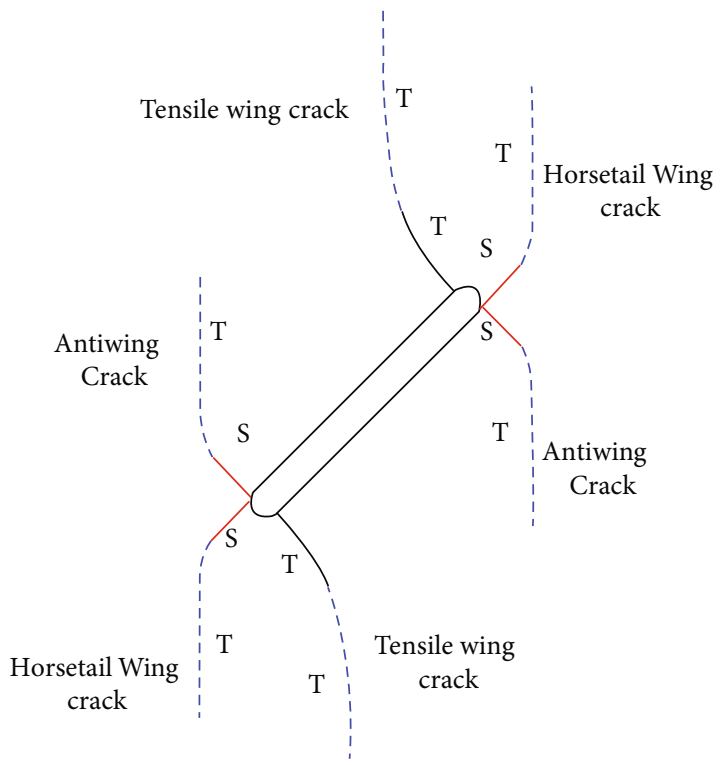

FIGURE 6: Schematic illustration of different types of crack (T stands for tensile crack, S stands for shear crack) [9].

and then shifted and spread toward the maximal stress and along the direction it went on with its propagation. After that, the antiwing and horsetail cracks almost appeared simultaneously. For filled joints, the crack initiation sequence was similar to that of unfilled joints. The wing crack appeared rather early, the horsetail followed. But in most of the models, the antiwing crack was not observed except in the models where the joint inclination angles were of $0^{\circ}$, $45^{\circ}$, and $75^{\circ}$, respectively.

3.1.2. Crack Property Identified. How to assess the dynamic mechanism of the crack? In physical experiments, the researchers usually make judgments by observing its surface smoothness and the presence of the crumbed bits. Those whose surfaces are smooth, clean, and bits free are described as tensile cracks. Those whose surfaces are rough, indented, and crumbed are described as shear cracks. In our paper, we will determine the type of crack failure (tensile or shear) in line with Reference [9], that is, by extracting the corresponding dynamic parameters such as the pressure at Gauss point, Mises stress, and yielding stress.

With tensile failures, as shown in Figure 8(a), the timelength curves for pressure, Mises stress, and yielding stress are characterized as follows:

(1) Before tensile failure or yielding, the pressure will be positive in value

(2) Before tensile failure or yielding, the Mises stress and the yielding stress both tend to decrease but the former decreases at a greater rate

(3) When the tensile crack is at the initiation stage, the Mises stress and the yielding stress will not necessarily decrease to zero

With shear failures, as shown in Figure 8(b), the timelength curves for pressure, Mises stress, and yielding stress are characterized as follows:

(1) Before shear failure or yielding, the pressure will be positive in value

(2) Before shear failure or yielding, the Mises stress and the yielding stress both tend to increase but the former increases at a greater rate

(3) When the shear crack is at the initiation stage, the pressure, the Mises stress, and the yielding stress will not necessarily decrease to zero

3.2. The Crack Initiation Position and Propagation Path. The initiation position and angle are two important indexes for the description of the crack breaking behavior. The wing crack initiation is usually induced by tensile failure, and it makes its appearance at the end or near the end of the joint. In Figure 9(a), the initiation positions for the wing cracks in the filled and unfilled specimens at different joint inclination angles are presented. It can be clearly seen from the figure that for filled and unfilled specimens, the initiation position of the wing crack is affected by the variation of the joint inclination angle. When the inclination angle is 00 , the initiation position of the wing crack is near the center of the joint. With the increase of the inclination angle, the initiation position will move gradually toward the round terminal zone. The differences between the filled and the unfilled specimens lie in that when the inclination angle is fairly small $\left(<30^{\circ}\right)$, the former's initiation position of the wing crack is nearer to the round terminal zone. The initiation position for the antiwing crack is similar to that for the wing crack, and generally, it can be located at or near the end of the joint, as can be seen in Figure 9(b). As can be seen clearly from the figure, the initiation position is also affected by the variation of the inclination angle. With the angle increasing, the initiation position of the antiwing crack will move from the end of the joint to its straight side.

To better describe how filling affects the crack prorogation path, in the present paper, we will turn to the wing crack initiation angle $\theta$ and the antiwing initiation angle $\gamma$. As can 

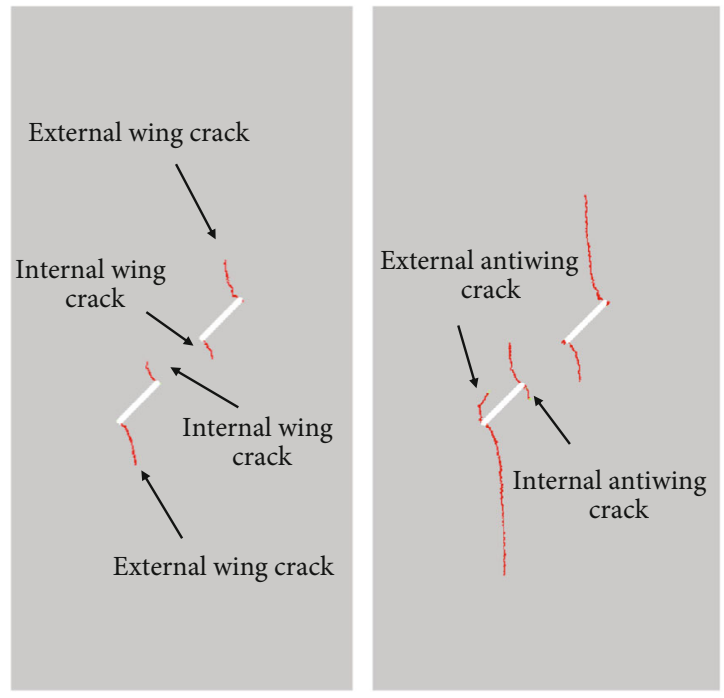

(a)
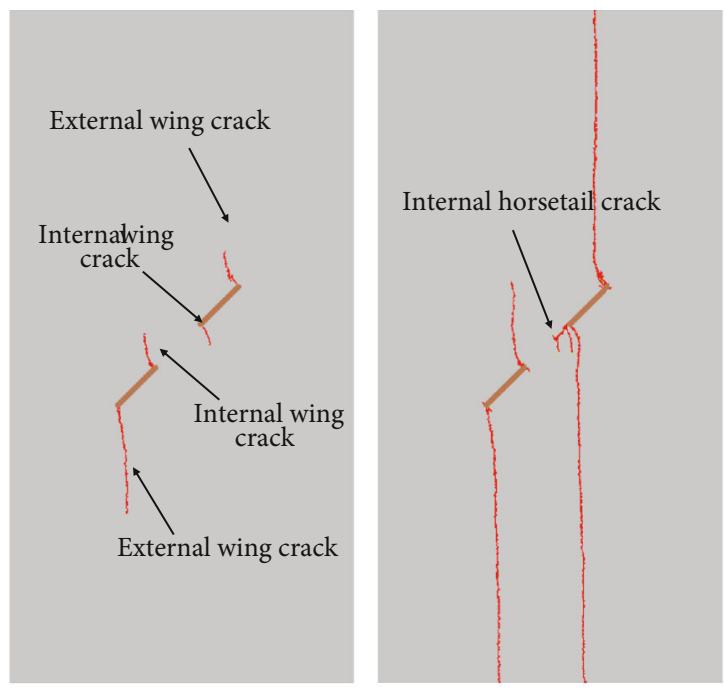

(b)
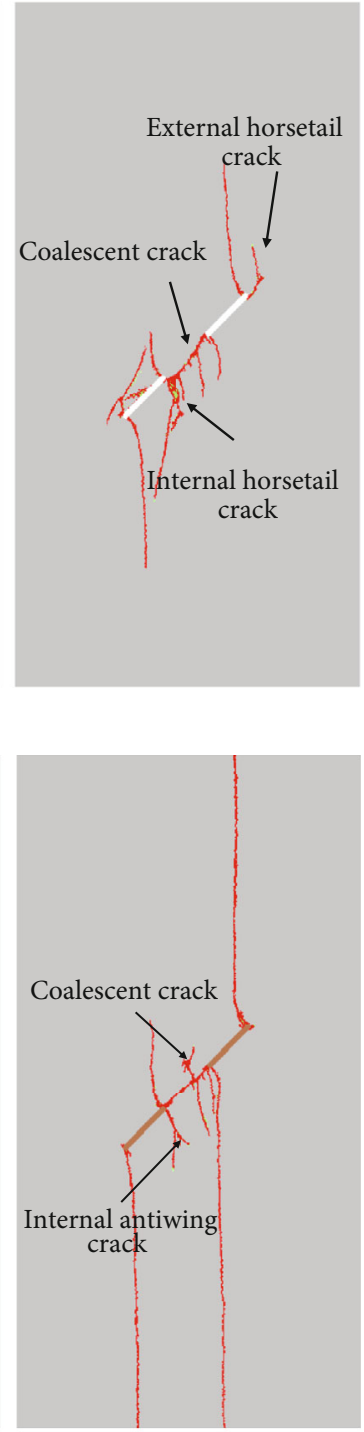

Figure 7: The prepared joint's main branching cracks and their propagation processes $\left(\beta=45^{\circ}\right)$. (a) Cracks unfilled. (b) Cracks filled.

be seen from Figure 10, the initiation angle of the tensile wing crack and that of the antiwing crack for the filled specimen is smaller than those for the unfilled specimen. In Figure 10(a), the initiation angles for the wing cracks in filled and unfilled specimens are presented. As can be seen from the figure, when the joint inclination angle is small $\left(<15^{\circ}\right)$, the crack initiation angle of the wing joint increases with the increase of the inclination angle. When the inclination angle continues to increase, the initiation angle of the wing crack will decrease. Figure 10(b) is about the initiation angle of the antiwing crack. Contrary to the findings with the wing crack, for antiwing cracks, the initiation angle increases with the increase of the joint inclination angle.

3.3. The Coalescence Mode of Crack. In the present paper, the coalescence modes of the noncoalescent cracks of the coplanes are studied against 7 joint inclination angles $\left(0^{\circ}\right.$, $15^{\circ}, 30^{\circ}, 45^{\circ}, 60^{\circ}, 75^{\circ}$, and $\left.90^{\circ}\right)$. With every inclination angle as an affecting factor, both filled and unfilled joints are studied. Therefore, 14 numerical models were established with the simulation results shown in Figure 11. Without a special statement, $\mathrm{S}$ and $\mathrm{T}$ in the figure refer to the shear and the tensile crack, respectively. The identification of the crack type was carried out by turning to the mechanical information of the cracks. And this point had been discussed previously and so will not be further discussed here. According to Reference [6], the joint coalescence modes were classified as direct coalescence, indirect coalescence, and noncoalescence. A case was regarded as direct coalescence when the coalescence point was near the reference line (see Figure 12(a)). Otherwise, it would be defined as indirect coalescence (see Figures 12(b) and 12(c)).

3.3.1. Direct Coalescence. Zhang et al. [14] are convinced that the direct coalescence can easily take place at the two internal ends of the prepared cracks. After damage, in the rock bridge 


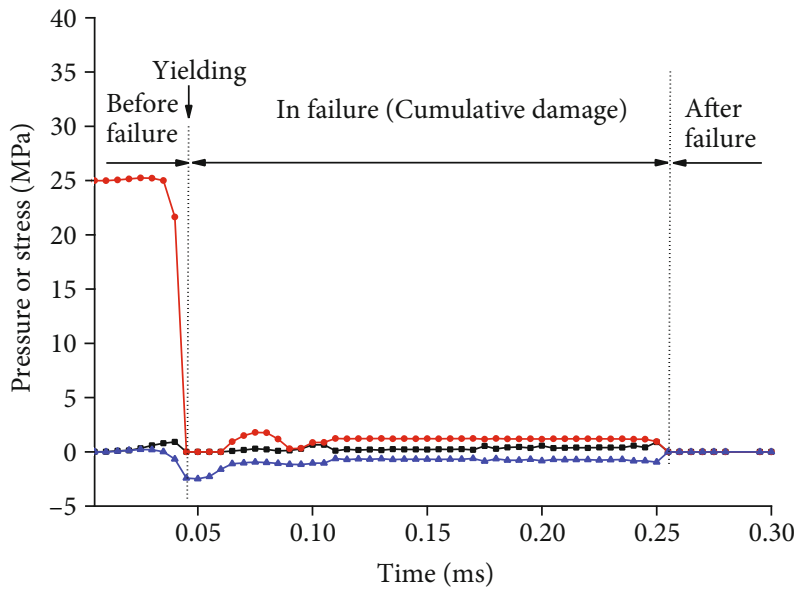

(a) Mechanic information for the tensile failure at Gauss point

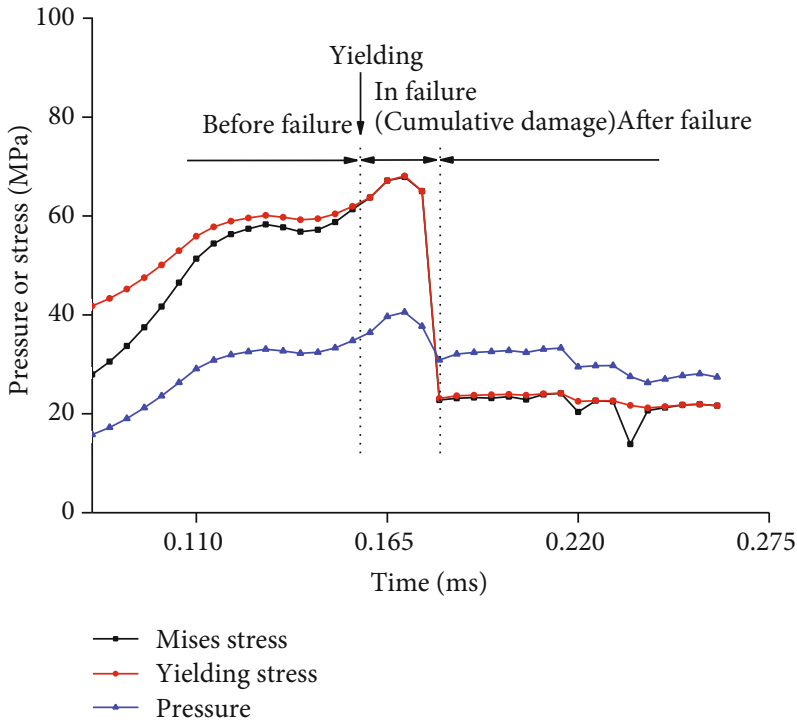

(b) The mechanical information for shear failure at Gauss point

FIGURE 8: Time-length curves for pressure, Mises stress, and yield stress.

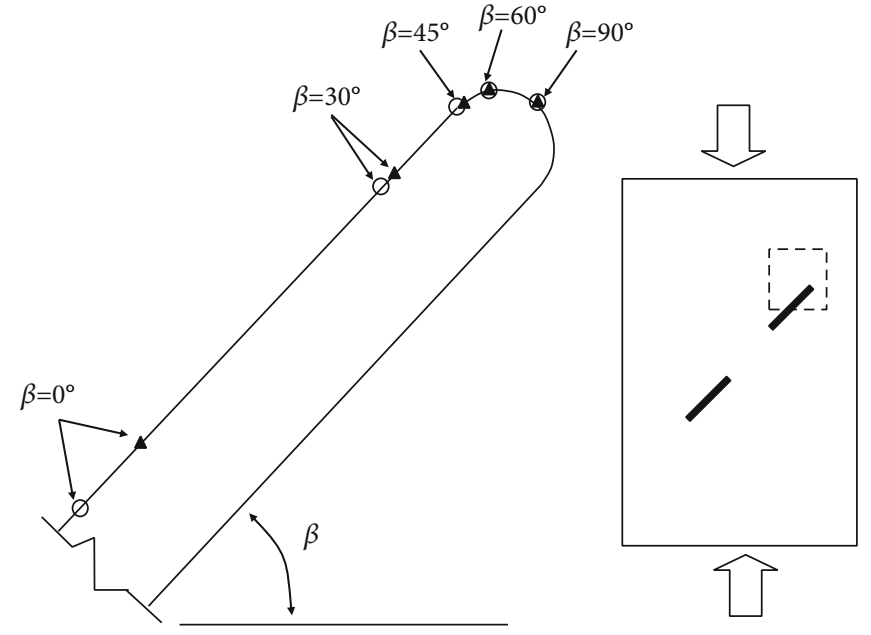

(a) An illustration of joint inclination angle as a function of initiation position of the tensile wing crack
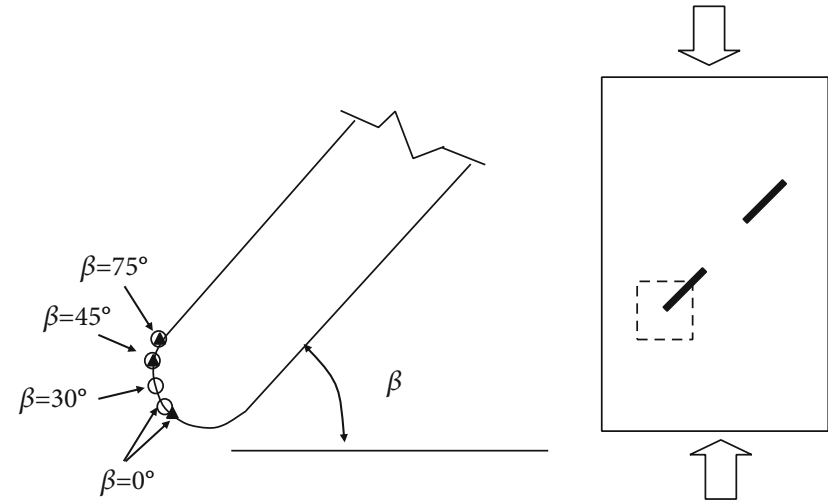

$\Delta$ Filled joint

$\bigcirc$ Unfilled joint

(b) Illustration of joint inclination angle as a function of flaw initiation position of antiwing crack

FIGURE 9: Illustration of joint inclination angle as a function of crack initiation position.

zone, a coplanar broken profile will appear, similar to the prepared crack. In the present paper, we obtained modes of direct coalescence as shown in Figure 11. From the figure, it can be seen that under high strain loading, the shear coalescence takes place only when the joint inclination angle varies within the range of $45^{\circ} \leq \beta \leq 60^{\circ}$. With unfilled joints, when the joint inclination angles are at $45^{\circ}$ and $60^{\circ}$, the direct shear coalescence takes place easily at the two ends of the prepared crack. With filled joints, the direct coalescence can take place at the internal end of the crack only when the joint inclination angle is at $45^{\circ}$. Taking into account the research results of Wong and $\mathrm{Li}$ [23], we believe that when the rock bridge is short $(L \leq 2 a)$ and the joint inclination angle varies within the medium range, the shear direct coalescence can occur when the crack angle comes to its completion.

In Figure 7, a detailed coalescence process is described which consists of crack initiation, propagation, and coalescence. As can be seen from the figure, when the direct shear coalescence crack occurs at the internal end, the tensile wing crack is already in the stage of stable propagation. In addition, the external wing crack is apparently longer than the internal wing crack. With the increase of the load, the shear 


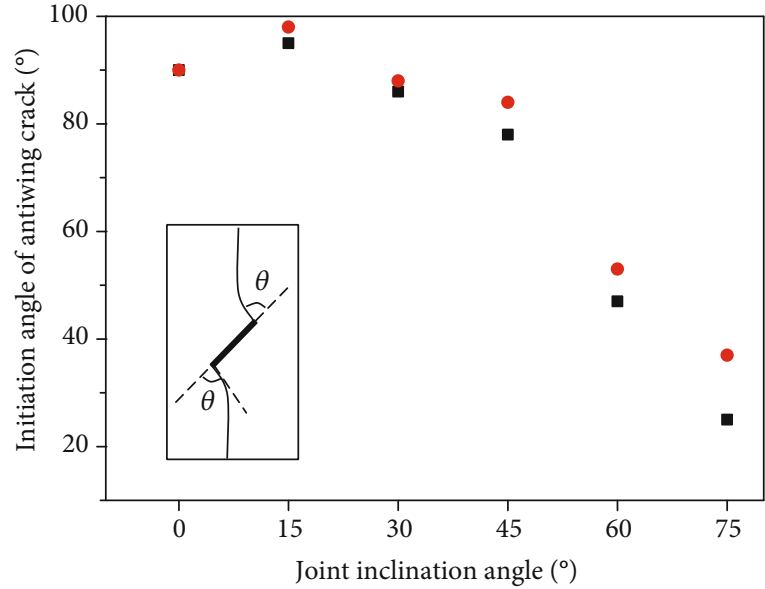

(a) Joint inclination angle as a function of the initiation angle of tensile wing crack

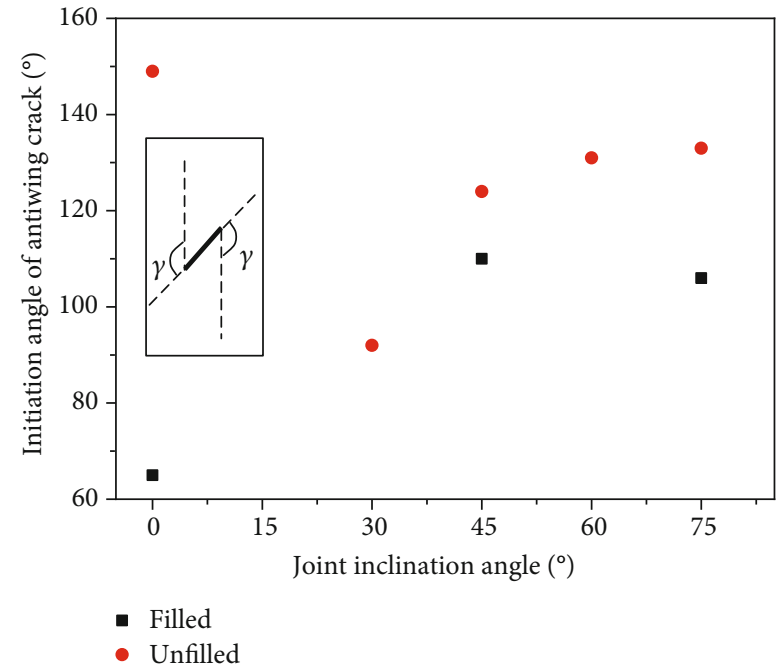

(b) Joint inclination angle as a function of initiation angle of antiwing crack

Figure 10: Joint inclination angle as a function of initiation angle of antiwing crack.

crack goes from one end of the prepared crack to the initiation point of the other end. And finally, the two internal initiation points of the internal crack will be connected, forming a macro coalescent broken band. Therefore, we conclude that the shear coalescence occurs mainly in the rock bridge zone.

3.3.2. Indirect Coalescence. Compared with the direct coalescence, in the numerical simulation, the indirect coalescences are more frequently witnessed. With the unfilled joint, when the inclination angle is small $\left(\beta \leq 30^{\circ}\right)$, a Y type of coalescence between rock bridges will be mainly induced by antiwing and horsetail wing. When the joint inclination angle is rather large $\left(\beta=75^{\circ}\right)$, in the rock bridge zone, the tensileshear coalescence will be induced by wing and antiwing cracks. And in all the above cases, only two cracks are involved. From Figure 11, it can be seen that with the filled joint, the indirect crack coalescence is more complicated, for the process generally involves 3 or more cracks. For example, when the inclination angle $\beta=15^{\circ}$ or $30^{\circ}$, in the rock bridge zone, the $\mathrm{N}$ type of tensile-shear compound coalescence will occur. When $\beta=60^{\circ}$, in the coalescence, more than 3 cracks are involved. In addition, the abovementioned modes cannot be used directly to describe the cracks thus produced. But considering the crack property, we can say that such compound indirect coalescence still belongs to the tensile-shear type. When $\beta=75^{\circ}$, the coalescence witnessed in the rock bridge zone is similar to that witnessed in the unfilled joint, that is, it will be of a tensile-shear type, induced by wing and antiwing cracks. Therefore, we can conclude that with an unfilled joint, the indirect coalescence of the crack is affected by the inclination angle. When the angle is small $\left(\beta \leq 30^{\circ}\right)$, in the rock bridge zone, under dynamic loading, the $\mathrm{Y}$ type of shear coalescence will occur. When the angle is large $\left(\beta=75^{\circ}\right)$, the tensile-shear compound coalescence will occur.
Filing has complicated the coalescence mode in the rock bridge zone, that is, the main mode will be the tensile-shear compound. But it is worth mentioning that when the inclination angle $\beta=0^{\circ}$ or $90^{\circ}$, it will not be easy for coalescence to occur at the internal end of the prepared crack.

3.4. Analysis of the Damage of the Joint Rock. In Figure 13, under the same loading, the damage to the filled specimen and that to the unfilled specimen are compared under the condition that $\beta=0^{\circ}$ or $90^{\circ}$. It is apparent from the figure that by uniaxial compression, under the same load, the damage to the filled specimen is less severe than that to the unfilled specimen, showing that the crack resistance of the filled specimen is greater than that of the unfilled specimen. This can be mainly attributed to the fact that filling has altered the stress distribution around the crack, leading to the diminishing of the stress value and narrowing of the distribution area. According to the maximal tensile theory of the fracture mechanics and the mechanical performance of the rock, we know that the decrease of the tensile stress at the tip of the crack has increased the crack resistance of the rock material [24].

\section{Conclusion}

By turning to numerical simulation, investigations were conducted into filling as a function of crack propagation under uniaxial dynamic loading. From such perspectives as the initiation position, sequence and angle, and coalescence mode, the crack behaviors of the filled and unfilled specimens are compared with conclusions drawn as follows:

(1) For the specimen, filled and unfilled, with the increase of the joint inclination angle, the initiation position of the tensile wing crack will move to the round end of the joint. When the inclination angle 


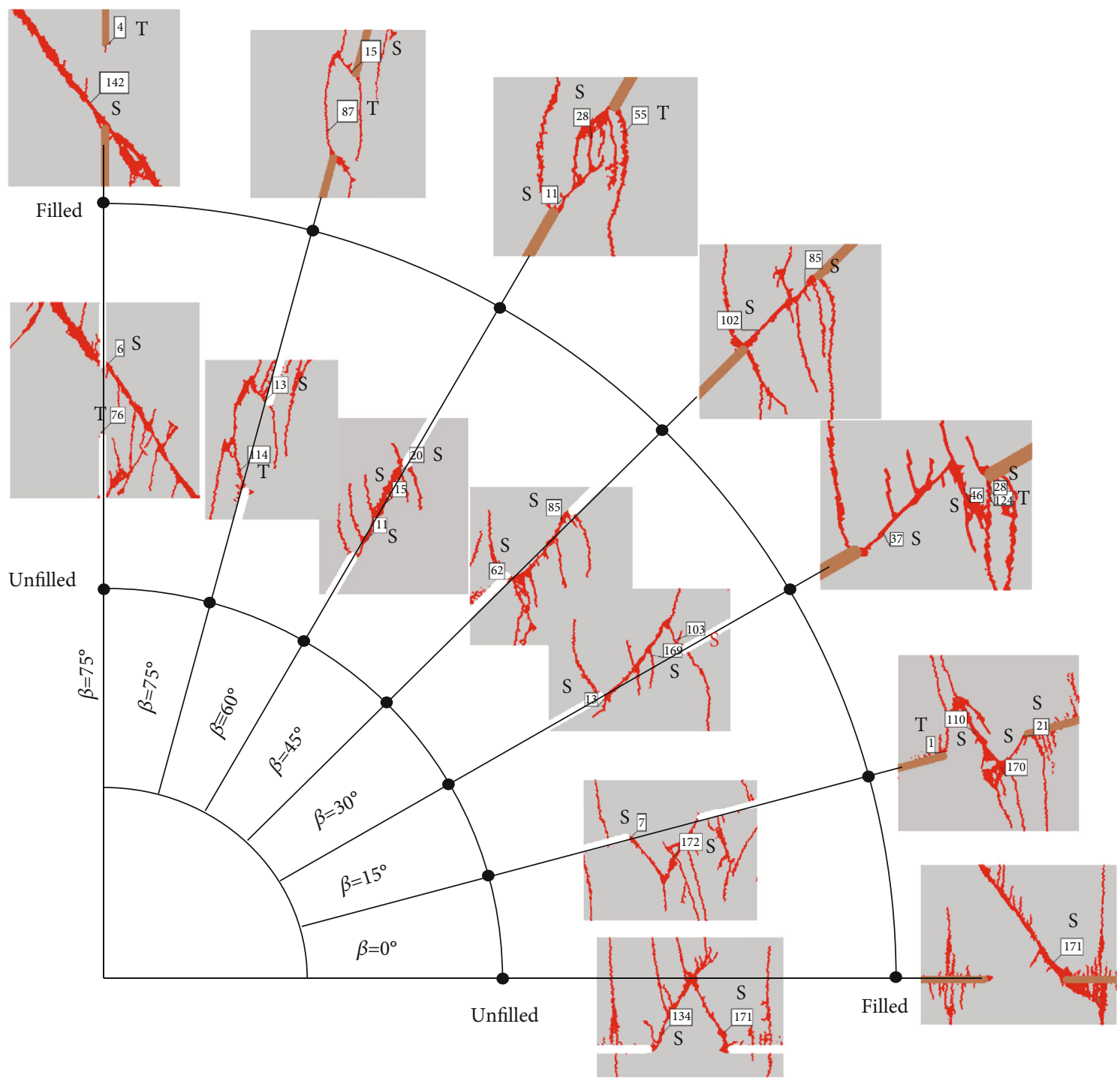

FIGURE 11: Joint inclination angle as a function of crack coalescence modes.

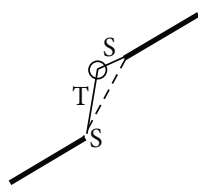

- - - Reference line in the internal end or the joint

○ Coalescence

(a)

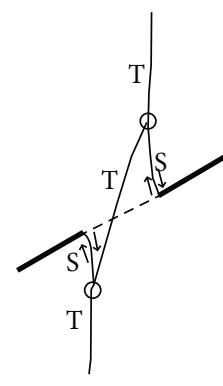

(b)

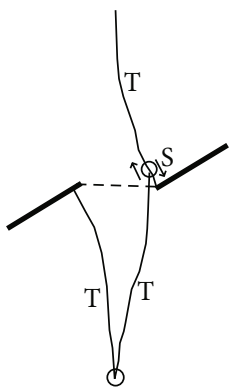

(c)
Figure 12: Distinctions between the direct and indirect coalescences [6].

is small $\left(\beta<30^{\circ}\right)$, the initiation position of the wing crack of the filled specimen is near the round end of the joint. The law governing the behavior of the initiation position of the antiwing crack runs contrary to the law governing the behavior of the initiation position of the tensile wing crack, and with the enlargement of the inclination angle, the initiation position of the crack will move to the straight side at the end of the joint

(2) With the filled specimen, the initiation angle of the tensile wing crack and that of the antiwing crack are smaller than that for the unfilled specimen

(3) Crack coalescence modes are affected by joint inclination angle and crack filling. Whether the crack of the specimen is filled or not, under uniaxial compression, only when the joint inclination angle varies in the medium range can the direct coalescence occur. And indirect coalescence can be more frequently witnessed than the direct coalescence in numerical simulation

(4) Under uniaxial compression, with the same loading, the specimen with cracks filled is damaged less severely than the specimen with cracks unfilled 


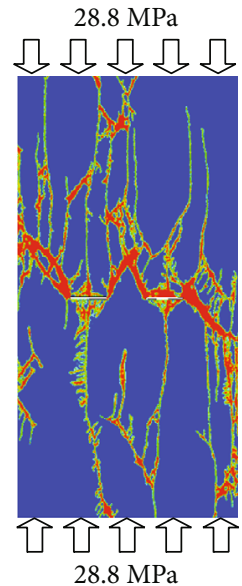

(a)

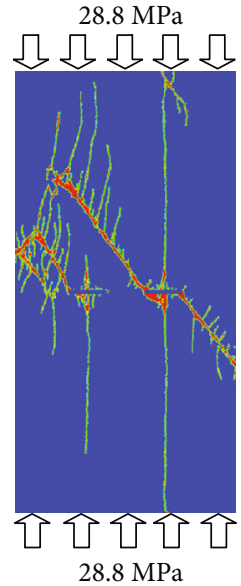

(b)

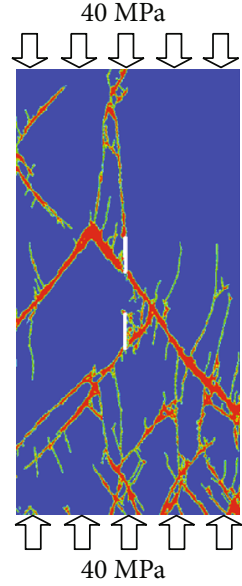

(c)

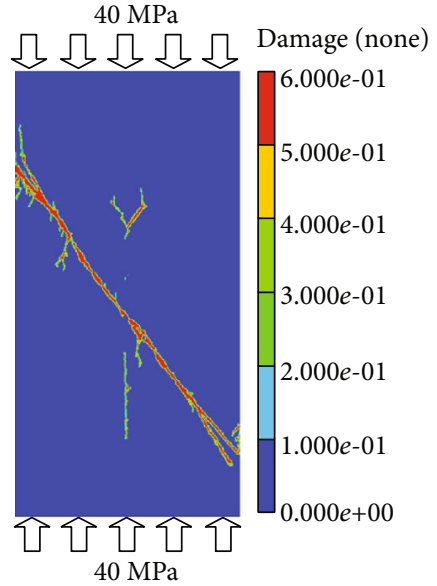

(d)

Figure 13: Damage distribution under different conditions. (a) With $\beta=0^{\circ}$, damage to unfilled specimen. (b) With $\beta=0^{\circ}$, damage to filled specimen. (c) With $\beta=90^{\circ}$, damage to unfilled specimen. (d) With $\beta=90^{\circ}$, damage to filled specimen.

\section{Data Availability}

The data used to support the findings of this study are available from the corresponding author upon request.

\section{Conflicts of Interest}

The authors declared no conflicts of interest.

\section{Acknowledgments}

This work was supported by the National Key Research and Development Project of China (2017YFC0603003), National Natural Science Foundation of China (51974009 and 51674008), Key Research and Development Program of Anhui Province (201904a07020010), Leading Talent Project of Anhui "Special Support Program", Anhui Provincial Academic and Technology Leaders Research Activities Funding (2018D187), Excellence Talent Training Program of High School (gxbjZD2016051), and Innovation Team Construction Project of Anhui High School Research Platform.

\section{References}

[1] H. P. Xie, M. Z. Gao, R. Zhang, G. Y. Peng, W. Y. Wang, and A. Q. Li, "Study on the mechanical properties and mechanical response of coal mining at $1000 \mathrm{~m}$ or deeper," Rock Mechanics and Rock Engineering, vol. 52, no. 5, pp. 1475-1490, 2019.

[2] Z. H. Chen, X. B. Li, W. Lei, S. F. Wang, and L. J. Dong, "Influence of flaw inclination angle on unloading responses of brittle rock in deep underground," Geofluids, vol. 2019, Article ID 4657645, 16 pages, 2019.

[3] P. Feng, F. Dai, Y. Liu, G. Y. Peng, and H. B. Du, "Mechanical behaviors of rock-like specimens with two non-coplanar fissures subjected to coupled static-dynamic loads," Engineering Fracture Mechanics, vol. 199, pp. 692-704, 2018.

[4] W. B. Lu, H. B. Li, M. Chen, C. B. Zhou, and X. X. Wu, "Several key problems with safe Vrbration judgement and application in hydropower engineering blasting," Chinese Journal of Rock
Mechanics and Engineering, vol. 28, no. 8, pp. 4180-4184, 2009.

[5] L. N. Y. Wong and H. H. Einstein, "Systematic evaluation of cracking behavior in specimens containing single flaws under uniaxial compression," International Journal of Rock Mechanics and Mining Sciences, vol. 46, no. 2, pp. 239-249, 2009.

[6] L. N. Y. Wong and H. H. Einstein, "Crack coalescence in molded gypsum and Carrara marble: part 1. Macroscopic observations and interpretation," Rock Mechanics and Rock Engineering, vol. 42, no. 3, pp. 475-511, 2009.

[7] M. Prudencio and M. V. S. Jan, "Strength and failure modes of rock mass models with non-persistent joints," International Journal of Rock Mechanics and Mining Sciences, vol. 44, no. 6, pp. 890-902, 2007.

[8] P. Wang, K. Li, C. W. Song, Y. J. Zhai, C. K. Zhao, and S. X. Xiao, "Numerical simulation of hole-crack propagation under uniaxial stress using in energy fracturing development technology," Fresenius Environmental Bulletin, vol. 28, no. 12, pp. 10154-10163, 2019.

[9] H. Q. Li and L. N. Y. Wong, "Numerical study on coalescence of pre-existing flaw pairs in rock-like material," Rock Mechanics and Rock Engineering, vol. 47, no. 6, pp. 2087-2105, 2014.

[10] G. W. Ma, X. M. An, and L. He, "The numerical manifold method: a review," International Journal of Computational Methods, vol. 7, no. 1, pp. 1-32, 2011.

[11] M. Bahaaddini, G. Sharrock, and B. K. Hebblewhite, "Numerical investigation of the effect of joint geometrical parameters on the mechanical properties of a non-persistent jointed rock mass under uniaxial compression," Computers and Geotechnics, vol. 49, pp. 206-225, 2013.

[12] X. B. Li, F. Q. Gong, M. Tao, L. J. Dong, K. Du, and C. D. Ma, "Failure mechanism and coupled static-dynamic loading theory in deep hard rock mining: a review," Journal of Rock Mechanics and Geotechnical Engineering, vol. 9, no. 4, pp. 767-782, 2017.

[13] L. L. Niu, W. C. Zhu, S. H. Li, S. Li, and M. Yu, "Experimental study of dynamic characteristics of sandstone under intermediate strain produced by pendulum hammering," Chinese Journal of Rock Mechanics and Engineering, vol. 33, no. 12, pp. 2443-2450, 2014. 
[14] P. Zhang, N. Li, R. L. He, and J. G. Xu, "Mechanism of fracture coalescence between two pre-existing flaws under dynamic loading," Chinese Journal of Rock Mechanics and Engineering, vol. 25, no. 6, pp. 1210-1217, 2006.

[15] X. P. Zhang and L. N. Y. Wong, "Loading rate effects on cracking behavior of flaw-contained specimens under uniaxial compression," International Journal of Fracture, vol. 180, no. 1, pp. 93-110, 2013.

[16] H. Lee and S. Jeon, "An experimental and numerical study of fracture coalescence in pre-cracked specimens under uniaxial compression," International Journal of Solids and Structures, vol. 48, no. 6, pp. 979-999, 2011.

[17] X. Chang, D. Yan, Z. H. Li, S. R. Wang, and C. A. Tang, "Crack propagation from a filled flaw in rocks considering the infill influences," Journal of Applied Geophysics, vol. 152, pp. 137149, 2018.

[18] A. A. Persson, "Simple model for the dynamic deformation and failure properties of brittle materials," in 4th Int. Symp. Ceramic Materials and Components for Engines Goteborg, vol. 10, no. 12, 1991Springer, 1991.

[19] Century Dynamic Inc, AUTODYN Theory Manual, Concord, CA: Century Dynamics, Inc., Concord California, 2003.

[20] A. Z. Zhu, B. Mohanty, and H. Xie, "Numerical investigation of blasting-induced crack initiation and propagation in rocks," International Journal of Rock Mechanics and Mining Sciences, vol. 44, no. 3, pp. 412-424, 2007.

[21] M. Wang, Z. M. Zhu, and J. Xie, "Experimental and numerical studies of the mixed-mode I and II crack propagation under dynamic loading using SFPB," Chinese Journal of Rock Mechanics and Engineering, vol. 34, no. 12, pp. 2474-2485, 2015.

[22] D. F. Cen, D. Huang, Y. X. Song, and Q. H. Jiang, "Direct tensile behavior of limestone and sandstone with bedding planes at different strain rates," Rock Mechanics and Rock Engineering, vol. 53, no. 6, pp. 2643-2651, 2020.

[23] L. N. Y. Wong and H. Q. Li, "Numerical study on coalescence of two pre-existing coplanar flaws in rock," International Journal of Solids and Structures, vol. 50, no. 22-23, pp. 3685-3706, 2013.

[24] B. Zhang, S. C. Li, D. F. Zhang, M. T. Li, and D. L. Shao, "Niaxial compression experiments with mechanical property and fracture of and damage to the material similar to the rock with joint crack filled," Rock and Soil Mechanics, vol. 33, no. 6, pp. 1647-1652, 2012. 